Rechtsmedizin 2014 · 24:245-246

DOI 10.1007/s00194-014-0972-3

Online publiziert: 26. Juli 2014

(c) Springer-Verlag Berlin Heidelberg 2014

\section{B. Bockholdt}

Institut für Rechtsmedizin, Universitätsmedizin Greifswald,

Ernst-Moritz-Arndt-Universität Greifswald

\section{Jahrestagung der Deutschen Gesellschaft für Rechtsmedizin}

\author{
Greifswald + Heringsdorf auf \\ Usedom, 09.09.-13.09.2014
}

Liebe Leserinnen und Leser,

wir freuen uns sehr, Sie zur 93. Jahrestagung der Deutschen Gesellschaft für Rechtsmedizin (DGRM) in Greifswald und Heringsdorf auf das Herzlichste willkommen heißen zu dürfen. Erstmals hat die Mitgliederversammlung der DGRM beschlossen, dass die Jahrestagung durch das Institut für Rechtsmedizin der Universitätsmedizin Greifswald ausgerichtet wird.

Das Institut für Rechtsmedizin Greifswald gehört zu den ältesten Instituten im deutschsprachigen Raum. Es hat sich als Teil der Universitätsmedizin in den letzten Jahren den wachsenden Anforderungen entsprechend, aber auch unter nichteinfachen allgemeinen Bedingungen nachhaltig positiv entwickelt.

Als eine der wesentlichen Aufgaben einer universitären Rechtsmedizin kommt für uns der akademischen Lehre, der festen Verankerung in der ärztlichen Fort- und Weiterbildung und in der Fortbildung für andere medizinische und nichtmedizinische Berufsgruppen ein hoher Stellenwert zu. Die seit Jahren von den Studierenden der Medizin, der Zahnmedizin, aber auch fakultätsübergreifend kontinuierlich als exzellent evaluierte rechtsmedizinische Lehre ist durchaus eine Anerkennung der Leistungen der Mitarbeiter des Instituts und hat in den letzten Jahren die Position der
Greifswalder Rechtsmedizin im universitären Fächerverbund wesentlich gefestigt.

Die Ernst-Moritz-Arndt-Universität wurde im Oktober 1456 in der Nikolaikirche (heute Dom St. Nikolai) gegründet. Die damalige medizinische Fakultät war eine der Gründungsfakultäten der Universität. In dieser Tradition stehend, ist es uns wichtig, die Eröffnungsveranstaltung unserer Jahrestagung im Greifswalder Dom durchzuführen. Da das Institut die Universitätsmedizin und den eher ländlich geprägten Nordosten der Bundesrepublik versorgt und wir die regionalen Bezüge zur Entwicklung des Fachs in den letzten Jahren zum Schwerpunktthema wählten, haben wir uns entschlossen, etwas abweichend zu der Tradition, in Heringsdorf auf der Insel Usedom in den wissenschaftlichen Austausch zu treten. So erhalten Sie einen kleinen Einblick in die Region, für die das Institut in seinen Versorgungsaufgaben zuständig ist.

\section{》) Vorträge und Poster-Prä- sentationen spiegeln die aktive, interdisziplinäre Forschung wider}

Die zahlreichen Vorträge und Poster-Präsentationen spiegeln die aktive und vermehrt interdisziplinäre Forschung, die einerseits die gesamte klassische Breite des Faches, andererseits aber v. a. auch die jüngst entwickelten Aufgaben der Rechtsmedizin wie die forensische Bild- gebung oder die Erweiterung der klinischen Rechtsmedizin wider. Die an vielen Instituten eingerichteten Untersuchungsstellen für Gewaltopfer erfordern natürlich eine wissenschaftliche Begleitung; nur so können die neuesten Erkenntnisse entsprechend umgesetzt werden. Zahlreiche Beiträge zeigen diese Entwicklung, belegen aber auch die regionalen Unterschiede, die gerade die Vielfalt dieses neuen Aufgabengebiets deutlich macht. Die über viele Jahre auch in Greifswald hervorragend entwickelte feste Verankerung, die die Rechtsmedizin in der Klinik und dabei ganz besonders im Kinderschutz erlangt hat, kennzeichnet ihre Interdisziplinarität, die notwendig ist, um weiterhin stark für die Zukunft zu sein. Da sich die Rechtsmedizin als wichtige Schnittstelle zwischen Medizin und Jurisprudenz versteht, ist es selbstverständlich, dass wir uns auch mit aktuellen Entwicklungen, so z. B. den Richtlinien zur Hirntoddiagnostik im wissenschaftlichen Kontext mit der aktuellen Diskussion, auseinandersetzen. Die rechtsmedizinische Expertise soll dabei die wissenschaftliche Diskussion bereichern.

Auch in den Bereichen der forensischen Toxikologie zeigen sich weitere Entwicklungen; die Liquid-Chromatographie-Massenspektrometrie/Massenspektrometrie (LC-MS/MS) ist inzwischen in der Analytik fest etabliert, und in der Fahreignungsbegutachtung spielt die forensische Toxikologie seit einigen 
Jahren eine wesentliche Rolle; die Intensität dürfte regional unterschiedlich sein. Die Forschungen auf dem Gebiet der forensischen Molekulargenetik führen da$\mathrm{zu}$, dass aus vergleichsweise ungünstigen Spuren immer besser die DNA extrahiert und untersucht werden kann. Auch die Bedeutung der RNA in der Forensik hat zugenommen und wird sich weiterentwickeln.

Zunehmend wird von den Studierenden der Medizin, auch in Greifswald, das Angebot von Famulaturen oder die Rechtsmedizin als Wahlfach im praktischen Jahr zu belegen, angenommen. Die Aneignung von Wissen, das nur die Rechtsmedizin systematisch vermittelt, wie beispielsweise zur ärztlichen Leichenschau oder zur Wundmorphologie und ihrer Dokumentation hat sich damit etabliert und bindet das Fach in die Universitätsmedizin ein. Es ist für uns selbstverständlich, dass Doktoranden betreut und Graduationen in den naturwissenschaftlichen Fächern angeboten werden. Um diese Aufgaben auch in Zukunft auf so hohem Niveau zu erbringen, ist natürlich eine gewisse Ausstattung der universitären Rechtsmedizin erforderlich. Die Forschungsleistungen, an denen die universitäre Rechtsmedizin auch in Zukunft stets gemessen werden wird, haben ihre Wurzeln häufig in den täglichen Versorgungsaufgaben. Beides trotz zunehmender Personalenge und Arbeitsverdichtung weiterhin auf hohem Niveau zu erfüllen und miteinander zu verbinden, bleibt eine der wichtigsten Herausforderungen in der Zukunft. Das International Journal of Legal Medicine, das im Fach meist zitierte Publikationsorgan, und die Rechtsmedizin stellen hervorragende Publikationsmöglichkeiten für unsere wissenschaftlichen Erkenntnisse dar.

Wir hoffen, dass mit dieser Jahrestagung, die auf großes internationales Interesse gestoßen ist, wieder einmal gezeigt werden kann, dass die deutschsprachige Rechtsmedizin ihre führende Position halten will und auch halten muss.

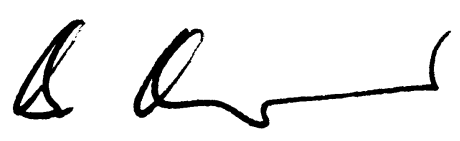

Prof. Dr. Britta Bockholdt

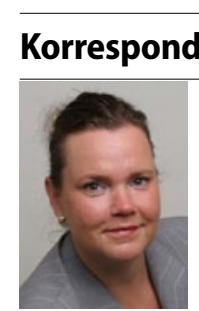

Institut für Rechtsmedizin, Universitätsmedizin

Greifswald, Ernst-Moritz-Arndt-

Universität Greifswald

Kuhstr. 30, 17489 Greifswald

britta.bockholdt@

uni-greifswald.de

\section{Einhaltung ethischer Richtlinien}

Interessenkonflikt. B. Bockholdt gibt an, dass kein Interessenkonflikt besteht.
M. Grassberger, H. Schmid Todesermittlung

Stuttgart: Wiss. Verlagsgesellschaft 2014, 2. Aufl., 379 S., 237 Abb.,

(ISBN 978-3-8047-3201-8), 59.00 EUR

Der Praxisleitfaden „Todesermittlung: Befundaufnahme und Spurensicherung" erscheint aktuell als 2. überarbeitete und ergänzte Auflage. Die Autoren Grassberger und Schmid legen mit ihrem Buch weit mehr als ein Kurzlehrbuch der Rechtsmedizin vor. In der Tradition der "Wiener Schule" stehend, geben sie dem Leser vielmehr einen Leitfaden der Tatortarbeit an die Hand, wobei sich das Buch nicht nur an den Rechtsmediziner, sondern auch an Polizeibeamte und andere Berufsgruppen richtet, die praktisch - also „vor Ort" - mit der Todesfallermittlung und -Bewertung befasst sind.

Die aktuelle Auflage behält die klare, übersichtliche thematische Gliederung der Erstausgabe, wobei die einzelnen Kapitel inhaltlich gestrafft und aktualisiert wurden. Nötige Korrekturen wurden, wo nötig, vorgenommen. Daneben wurde das Werk unter anderem um die Themen alpine Unfälle und verschiedene Arten des Erstickungstodes ergänzt.

Neben den rechtsmedizinischen Grundlagen der Thanatologie und forensischen Traumatologie, die in keinem Lehrbuch fehlen dürfen, wird dem Verhalten und dem strukturierten, spurenschonenden Vorgehen am Leichenfundort ein breiter Raum eingeräumt. Hier werden kriminalistische Aspekte und Techniken vorgestellt, die vor allem denjenigen wertvolle Ergänzung und Hilfe sind, die keine fundierte kriminalpolizeiliche Ausbildung genossen haben. Im umfangreichen Anhang sind, wie bereits von der ersten Ausgabe gewohnt, zahlreiche Schemata zur Befundund Spurensicherung, zur Bestimmung der Leichenliegezeit und zur Erstellung eines (kriminalpolizeilichen) Tatortbefundberichtes enthalten. Insgesamt handelt es sich um ein umfassendes, aktuelles Buch „aus der Praxis und für die Praxis".

T. Kamphausen (Köln) 